\title{
A catalog of the types of Elateridae (Coleoptera) deposited in the Museo Argentino de Ciencias Naturales, Buenos Aires
}

\author{
Axel O. BACHMANN ${ }^{1,2}$, Marta E. GUZMÁN de TOMÉ ${ }^{3} \&$ Mariel E. GUALA ${ }^{1}$ \\ ${ }^{1}$ División Entomología, Museo Argentino de Ciencias Naturales, Av. A. Gallardo 470, Buenos Aires, Argentina \\ C1405DJR. ${ }^{2}$ Departamento de Biodiversidad y Biología Experimental, Facultad de Ciencias Exactas y \\ Naturales, Universidad de Buenos Aires, Ciudad Universitaria, Buenos Aires, Argentina C1428EHA. \\ bachmann@bg.fcen.uba.ar ${ }^{3}$ Instituto Superior de Entomología, Facultad de Ciencias Naturales e Inst. M. Lillo \\ 205, 4000. Tucumán, Argentina. martaeguzman@gmail.com
}

\begin{abstract}
The type specimens (all current categories) of Elateridae deposited in this Museum are catalogued: twenty-four names are recorded. The specific names are alphabetically entered a single list, followed by the generic ones in their original spellings. An account of the types actually here deposited follows, with a description of all the labels. Later combinations, and synonyms are mentioned insofar they are known to the authors. In order to assist in future research, two further lists are added: 1 . of specimens labeled as types of names not found in the bibliography, and probably not published, and 2. of specimens labeled as types but not originally included in a type series, published or not after the original description (mainly 'allotypes').
\end{abstract}

Keywords: Type specimens, Elateridae, Coleoptera, Insecta.

Resumen: Catálogo de los tipos de Elateridae (Insecta, Coleoptera) depositados en el Museo Argentino de Ciencias Naturales, Buenos Aires. Se catalogan los ejemplares tipo de todas las categorías aceptadas de Elateridae depositados en este museo. Se registran veinticuatro nombres. Los nombres específicos se ordenan alfabéticamente; a estos siguen los genéricos, con la grafía original. Sigue la enumeración de los tipos efectivamente aquí presentes, con descripción de todos sus rótulos. Se mencionan las combinaciones ulteriores, y los sinónimos cuando son conocidos por los autores. Para facilitar futuras investigaciones, se agregan otras dos listas: 1. de ejemplares rotulados como tipos, de nombres no hallados en la bibliografía y probablemente no publicados, y 2. de los ejemplares rotulados como tipos, pero no incluidos originalmente en una serie típica, publicados o no después de la descripción original (principalmente 'alotipos').

Palabras clave: Ejemplares tipo, Elateridae, Coleoptera, Insecta.

\section{INTRODUCTION}

A catalog of the type specimens (all current categories) of Elateridae housed in the Entomological Division of this Museum is presented, referred to twenty-one available names; the specific names are alphabetically entered in a single series, each one being followed by the generic one, all with the original spellings; a brief bibliographic quoting follows, as well as a list of the specimens examinated, if these were mentioned. An account of the type specimens actually here housed follows, with a description of all the labels. In geographic references, if the country is not mentioned, Argentina is meant. Later nomenclatural or taxionomical changes are added, insofar they are known by the authors. Some comments are added in a note, as well as the current subfamilial and tribal assignation.

Most of the species dealt with were described by Schwarz (1896, 1900, 1904, 1906), some others by Burmeister (1875), Candèze (1896), Brèthes (1920) and Golbach $(1979,1987)$. The systematic and nomenclatural updating follows Bouchard et al. 2011.

For the identification of types not formally designated in the original publication, evidences from the labels or from the specimens are considered (art. 72.4 of the International Code of Zoological Nomenclature, 1999). If no types were 
designated, and the amount of specimens examinated was not stated, it is assumed that they are a series of syntypes (recommendation $73 \mathrm{~F}$ of the Code), sometimes 'sole syntypes'. Some specimens in the collection are labeled as types of names not found in the literature, and assumed not to have been published. These names are probably not available in the sense of the Code; however, in order to assist in future research, they conform a separate list. Specimens labeled as types, but not included in the original publication, and specimens designated as types later, published or not, cannot be accepted as pertaining to the type series; however, also in order to assist in future research, they conform a further, separate list.

In his collection, Carlos Bruch used to copy on own labels (most of them with a red frame, a few with a green frame) the names of the species described by other authors, very often without adding an original label. Some specimens in the Bruch collection are labeled 'Cotypus': we include them as syntypes or as paratypes, depending on the context. All the specimens labeled as types bear a small inverted label, as "MACN-En 0000", referred to the data base of the collection of the Museum, managed after "Aurora" (Rodríguez 2007-2011).

This Museum is identified with MACN. Other repositories are identified as follows: CBC, Carlos Bruch coll, in MACN; IFML, Instituto Fundación Miguel Lillo, Tucumán, HB, Hermann Burmeister coll, in MACN.

\section{CATALOG OF THE TYPES}

andinus [Pomachilius]. Brèthes 1920: 36. Perú: Chanchamayo, leg. Vigil.

One synt. on a card, remounted (it has a pinhole), "C.R. Vigil Pérou" handwr. on grayish paper; "Repreparo / M. Viana / I-1960" handwr.; "Pomachilius / andinus / Brèthes" handwr. by Brèthes. We add "Typus" print. on red paper. "MACN-En / 8324" print., inverted.

Notes: The spec. bears no typus label. Record omited by Schenkling (1927), and included in the Corrigenda by Blackwelder (1947: 1407). Currently in Elaterinae, Pomachiliini.

apicatus [Pomachilius]. Schwarz 1904: 73-74. Brasil, two spec. from Bruch.

One synt. on a card, "Rep. Brasil / 14.XI.1901 / C. Bruch" print., date handwr., black frame; "Typus" print. on pale green paper; "Pomachilius / apicatus / 25 n. sp. Schw." handwr. by Bruch, red frame. "MACN-En / 8348" print., inverted. Notes: Labeled only by Bruch, not by Schwarz. Recorded as Pomachilius apicatus by Golbach (1994: 41). Ex CBC. Currently in Elaterinae, Pomachiliini.

argentinus [Conoderus]. Golbach 1987: 18-20, f. A. Tucumán: Trancas: Trancas 29.I.1982 holot., Tucumán. Burruyacú, Siete de Abril, represa, 10.I.1982, in IFML; ten parat. in MACN; key to species.

Nine parat. on pointed cards, on five pins of two spec. each (one spec. lost) "R. Argentina / Tucuman. Dto. / Burruyacú - Chilcas / 10-25. XI.1979 / Col. R. Golbach" print.; all labels duplicated on each pin. Four parat. on rectangular transparent cards, one of them "R. Argentina / Tucuman / Dpto Burruyacú / Dique Las Colas / 8.I.1982 / Col. R. Golbach" print.; two of them "R. Argentina / Tucuman / Depto. Trancas / 29.I.1982" print.; one of them "R. Argentina / Santiago del / Estero - El Remate / 11.I.1982 Col. R.Golbach" print. All nine: "Paratipo" print. on yellow paper, "Conoderus [print.] / argentinus [handwr.] / Det. R. Golbach [print.]", "MACN-En / 8356 [through 8364]" print., inverted.

Note: Recorded as Conoderus argentinus by Guzmán de Tomé \& Aranda (2008: 571). Currently in Agrypninae, Oophorini.

atomus [Cosmesus]. Schwarz 1904: 80. Argentina: Buenos Aires?, two spec. from Bruch.

One synt.? on a card, "Rep. Argentina / Prov. Tucumán / IV.1897 / C. Bruch" print., date handwr.; "Typus" print. on pale green paper; "Cosmesus / atomus / typ. Schw." handwr. by Bruch, red frame. "MACN-En / 8343" print., inverted.

Notes: Labeled only by Bruch, not by Schwarz. The localities differ, although Schwarz put a question mark to Buenos Aires; otherwise the labels seem authentic. Mentioned under Mesembria by Golbach in litteris 21.VIII.1991, and so recorded by him (1994: 38). Ex CBC. Currently in Elaterinae, Elaterini.

bruchi (sub Bruchi) [Grammophorus (sic, pro Grammephorus ]. Schwarz, 1904: 71-72. Argentina: Neuquen Prov., two spec. (a couple?) from Bruch, the sole Argentine species.

bruchi [Duretia]: Golbach 1984: 42. Type species by monotypy.

One synt. pinned, "Rep. Argentina / Gob. 
Neuquen / 2.1898 / C. Bruch" print., date handwr., black frame; "Typus" print. on pale green paper; "Grammophorus / Bruchi / n. sp. 32 Schw." handwr. by Bruch, red frame. "MACN-En / 8340" print., inverted.

Notes: Labeled only by Bruch, not by Schwarz. Transferred to Duretia n. gen., as the type species by Golbach (1984: 42); recorded as Duretia bruchi by Golbach (1994: 31).

Ex CBC. Currently in Elaterinae, Elaterini.

bruchi (sub Bruchi) [Monadicus]. Schwarz 1906: 142-143. Buenos Aires, several spec. from Bruch.

One synt. on a card, "Rep. Argentina / Prov. BuenosAires / XII.1897 / C. Bruch” print., date handwr., black frame; "Schwarz det." print.; "Typus" print. on pale green paper; "Monadicus / Bruchi / tipo / Schw." handwr. by Bruch, red frame. "MACN-En / 8350" print., inverted.

Notes: Labeled only by Bruch, not by Schwarz. Recorded under Monadicusi by Golbach (1994: 39). Ex CBC. Currently in Negastriinae, Negastriini.

bruchi (sub Bruchi) [Pyrophorus]. Candèze 1896: 66. Not seen; Córdoba, fide Zool. Rec.

bruchi (sub Bruchi) [Pyrophorus]: Bruch 1911: 250. Collected in Chaco; Córdoba is an error.

bruchi [Fulgeochlizus]: Costa 1975: 103, f. 125128, 172. Type species of the new genus.

One synt. pinned, "Rep. Argentina / Gob. Chaco / 2.1895 / C. Bruch" print., date handwr., black frame; "Typus" print. on pale green paper; "Pyrophorus / Bruchi / Cand." handwr. by Bruch, red frame. "MACN-En / 8354" print., inverted.

Notes: Labeled only by Bruch, not by Candèze. Transferred to Fulgeochlizus n. gen. as the type species, and synonymized with Pyrophorus lineatocollis Schwarz 1906, by Costa (1975: 103, f. 125-128, 172). Recorded under Fulgeochlizus by Guzmán de Tomé \& Aranda (2008; 571). Ex CBC. Currently in Agrypninae, Pyrophorini.

cosmesoides [Parapomachilius]. Schwarz 1906: 140-141. Mendoza, several spec., leg. JensenHaarup.

One synt. on a card, "Jensen / Haarup" handwr.; "Rep. Argentina / Prov. Mendoza / 190_ / C. Bruch" print., black frame; "Cotypus" print. on pale green paper; "Parapomachilius / cosmesoides / n. sp. Schw." handwr. by Bruch, remainings of a black frame. "MACN-En / 8355" print., inverted.

Notes: Labeled only by Bruch, not by Schwarz.
Currently in Cosmesus Eschscholtz 1829, fide Arnett (1955: 601) (Parapomachilius Schwarz 1900 is a synonym). Recorded as Cosmesus cosmesoides by Golbach (1994: 41) and Lawrence (2001). Ex CBC. Currently in Elaterinae, Elaterini.

costae [Cardiorhinus]. Golbach 1983: 160, 162163, pl. II: 8, 9. Misiones Prov.

One parat. on a card, remounted (it has a pinhole), "Misiones / Bompland" print., remainings of a black frame; "Col. / C. Bruch" print., black frame; "Cardiorhinus [print.] / costae / n. sp. [handwr.] / det. R. Golbach 1981 [print., date handwr.]" on white paper; "Paratipo" print. on yellow paper. "MACN-En / 8736" print., inverted.

Note: Ex CBC. Currently in Elaterinae, Cardiorhinini.

crassiusculus [Ischiodontus]. Schwarz 1906: 106-107. Argentina.

One synt. on a card, "Rep. Argentina / Prov. Tucuman / I.1904 / C. Bruch" print., date handwr., black frame; "Cotypus" print. on pale green paper; "Ischiodontus / crassiusculus / Schw." handwr. by Bruch, black frame. "MACN-En / 8336" print., inverted.

Notes: Labeled only by Bruch, not by Schwarz. Currently in Dipropus, fide Arnett (1962: 9) (Ischiodontus Candèze 1859 is a synonym). Recorded as Dipropus crassiusculus by Golbach (1994: 37) and Lawrence (2001). Ex CBC. Currently in Elaterinae Dicrepidiini.

cruciger [Cosmesus] Schwarz 1904: 79. Buenos Aires, two $\hat{\partial}$, one $q$ from Bruch.

Three synt. side by side on one wide card, "Rep. Argentina / Prov. Buenos Aires / 4.1899 / C. Bruch" print., date handwr., black frame; "Typus" print. on pale green paper; "Cosmesus / cruziger / 32. n. sp. Schw." handwr. by Bruch, red frame. "MACN-En / 8344 [8345, 8346]" print., inverted.

Notes: Labeled only by Bruch, not by Schwarz. Synonymized with Cosmesus flavovittatus Schwarz 1900 by Bruch (1911: 254), synonymy not accepted by Golbach, personal communication (in litteris 21.VIII.1991) nor by Golbach (1994: 38); Arnett (1955: 601) puts Cosmesus as a junior synonym of Mesembria. Ex CBC. Currently in Elaterinae Elaterini.

decorus [Monocrepidius]. Burmeister 1875: 267. [Entre Ríos Prov.:] Paraná, dec. 1858, only once on Umbelliferae. 
Five synt. pinned, $1^{\text {st }}, 2^{₫}, 4^{\text {th }}$, and $5^{\text {th }}$ ones without labels, $3 ₫$ one "Parana. / Dec" print. on green paper. We add "Monocrepidius / decorus / Burmeister 1875 / Syntypus?". To the $4^{\text {th }}$ same but "Syntypus? / Typus?" handwr. on red paper. "MACN-En / 8424 [8425, 8426, 8427, 8428]" print. inverted.

Notes: Recorded under Conoderus by Fleutiaux (1911: 252), Schenkling (1925: 114), Blackwelder (1944: 287) and Golbach (1994: 34), because of priority over Monocrepidius. Handwr. labels from Burmeister not found on the bottom of the drawer.

Ex HB. Currently in Agrypninae Oophorini Monocrepidiina.

elegantulus [Aeolus]. Burmeister 1875: 268. "Aus hiesiger Gegend" (from here around), one spec.

Holot. pinned, "Buen. / Aires" print. on green paper. We add "Holotypus" print. on red paper, and "Aeolus / elegantulus / Burmeister 1875 / Holotypus", handwr. on red paper. "MACN-En / 8429" print., inverted.

Notes: Recorded as Aeolus elegantulus by Schenkling (1925: 122), Blackwelder (1944: 289), and Golbach (1994: 33). Handwr. labels from Burmeister (originally fastened on the bottom of the drawer) not found. Ex HB. Currently in Agrypninae Oophorini.

\section{ferrugineomarginatus [Monocrepidius].} Brèthes 1920: 36-37. Perú: Chanchamayo, Vigil leg.

One synt.? on a card , remounted (it has a pinhole), "C.R. Vigil - Pérou" handwr. on grayish paper; "Repreparo / M. Viana / I-1960" handwr.; "Monocrepidius / ferrugineomarginatus Br." handwr. by Brèthes. We add "Typus" print. on red paper. "MACN-En / 8325" print., inverted.

Notes: The spec. has no typus label, but otherwise it seems authentic. Recorded under Conoderus by Fleutiaux (1911: 252), Blackwelder (1944: 287) and Golbach (personal communication in litteris 21.VIII.1991), because of priority of Conoderus over Monocrepidius. Currently in Agrypninae Oophorini Monocrepidiina.

fulvescens [Pomachilius]. Schwarz 1904: 73. Buenos Aires, two spec. from Bruch, = Megapenthes longicollis Candèze in litteris.

One synt. on a card, "Rep. Argentina / Prov. Buenos Aires / 12.1897 / C. Bruch" print., date handwr., black frame; "Typus" print. on pale green paper; "Pomachilius / fulvescens / 24. n. sp.
Schw." handwr. by Bruch, red frame. "MACN-En / 8349" print., inverted.

Notes: Labeled only by Bruch, not by Schwarz. Recorded as Pomachilius fulvescens by Blackwelder (1944: 1407) and by Golbach (1994: 41). Ex CBC. Currently in Elaterinae Pomachiliini.

latifasciatus [Aeolus]. Schwarz 1906: 124. Brasil: Goyaz: Jatahy; with var. ruficollis.

One synt. on a card, "Jatahy / (Goyaz)" print.; "Schwarz det." print; "Cotypus" print. on pale green paper; "Aeolus / latifasciatus / n. sp. Schw." handwr. by Bruch, red frame. "MACN-En / 8330" print., inverted.

Notes: Labeled only by Bruch, not by Schwarz. Recorded as Aeolus latifasciatus by Blackwelder (1944: 290) and by Golbach, personal communication in litteris 21.VIII. 1991 Ex CBC. Currently in Agrypninae Oophorini Monocrepidiina.

latus [Horistonotus]. Golbach 1979: 412-414, f. 1-4. La Rioja Prov.: Iliar, km 128, 27487, leg. M. Gómez, holot. $\delta$ in MACN; Santiago del Estero Prov.: Los Tigres, allot. ㅇ Tucumán Prov., and parat. in IFML.

Holot. ô pinned, "La Rioja / Iliar / 1928 Gómez" handwr. on green paper, reverse white; "27487" handwr., red frame; " $>$ " print.; "Holotipo" print. on red paper; "Horistonotus / latus / n. sp. [handwr.] / R. Golbach det. [print.]" on white paper. "MACN-En / 8326" print., inverted. Currently in Cardiophorinae Cardiophorini.

luteus [Horistonotus]. Schwarz 1906: 145-146. Mendoza, several spec. Jensen-Haarup leg.

Two synt. on cards, on one pin, "Jensen / Haarup" handwr.; "Rep. Argentina / Prov. Mendoza / 190 / C. Bruch" print., black frame; "Cotypus" print. on pale green paper; "Horistonotus / luteus / Schw." handwr. by Bruch, remainings of a black frame. "MACN-En / 8352 [8353]" print., inverted.

Notes: Labeled only by Bruch, not by Schwarz. Recorded as Horistonotus luteus by Blackwelder (1944: 302), and by Golbach (1994:32). Ex CBC. Currently in Cardiophorinae Cardiophorini.

murinus [Stibadoderus]. Burmeister 1875: 272. Southern Patagonia: Santa Cruz Stream. murinus [Stibadoderus]: Golbach 1991: 84-86, f. 1-5. Redescription of the types.

Two synt. pinned, (one of them with part of an antenna on a card), " 54 " print. on green paper; "S. Cruz / Patag." print. on green paper; "Holotipo" 
["Allotipo"] print. on red paper; "Stibadoderus / murinus Burm. [handwr.] / R.Golbach det.1988 [print.]" on white paper. We add "Stibadoderus / murinus / Burmeister 1875 / Syntypus" handwr. on red paper. "MACN-En / 8430 [8431]" print., inverted.

Notes: As no type, nor amount of spec. were mentioned, the labels "Holotipo" and "Allotipo" are puzzling. Recorded as Stibadoderus murinus by Schenkling (1925: 195), Blackwelder (1944: 1408), and Golbach (1992: 84-86, f. 1-4). Ex HB. Currently in Elaterinae Pomachiliini.

mutabilis [Anoplischius]. Schwarz 1904: 51-52. South America, two $\delta$, three ${ }^{\circ}$, all different coloured.

Two synt. pinned, "Rep. Argentina / Prov. Buenos Aires / 4.1899 [1.1900] / C. Bruch" print., dates handwr., black frame; "Typus" print. on pale green paper; "Anoplischius / mutabilis / typ. [n. sp.] Schw." handwr. by Bruch, red frame. "MACN-En / 8333 [8334]" print., inverted.

Notes: Labeled only by Bruch, not by Schwarz. Recorded as Anoplischius mutabilis by Blackwelder (1944: 298), and by Golbach (1994: 36). Ex CBC. Currently in Elaterinae Dicrepidiini.

testaceipennis [Atractosomus]. Schwarz 1904: 70. Brasil, from Bruch.

One synt. on a card, "Rep. Brasil / XI.1901 / C. Bruch" print., date handwr., black frame; "Typus" print. on pale green paper; "Atractosomus / testaceipennis / typ. Schw." handwr. by Bruch, red frame. "MACN-En / 8335" print., inverted.

Notes: Labeled only by Bruch, not by Schwarz. Recorded as Atractosomus testaceipennis by Blackwelder (1944: 299), by Golbach (personal communication in litteris 21.VIII.1991) and Casari (2008). Ex CBC. Currently in Elaterinae Ampedini Dicrepidiina.

testaceus [Ischiodontus]. Schwarz 1904: 56-57. "Columbia"

One synt.? on a card, "Rep. Argentina / Prov. Buenos Aires / 9.VII.1903 / C. Bruch" print., date handwr., black frame; "Typus" print. on pale green paper; "Ischiodontus / testaceus / typ. Schw." handwr. by Bruch, red frame. "MACN-En / 8337" print., inverted.

Notes: Labeled only by Bruch, not by Schwarz. The label "Columbia" is puzzling. Recorded as Ischiodontus testaceus by Blackwelder (1944: 300), and under Dipropus by Arnett (1962: 9), Golbach (1994: 37), and M.Guzmán de Tomé
\& S. Aranda (2008: 572) because of priority of Dipropus over Ischiodontus. Ex CBC. Currently in Elaterinae Dicrepidiini.

\section{Types deemed to be housed in the museum but not found}

fueguensis [Athous]. Golbach \& Aranda 1991: 83-87, f. 1-7. Tierra del Fuego, leg. Sobral. Holot., allot., parat. in IFML, parat. in several repositories, including MACN.

Note: Parat. not found.

\section{Specimens labeled as types but not found in the bibliography, and probably not published}

\section{asperatus [Cardiorhinus]. Golbach}

One spec. pinned, "Argentina / Misiones" print., black frame; "38773" handwr., red frame; "Cardiorhinus / asperatus / n. sp. [handwr.] / R. Golbach det. 82 [print., date handwr.]" on white paper, black frame; "Paratipo" print. on yellow paper. "MACN-En / 8734" print., inverted. Ex CBC.

Notes: Probably not published. Not recorded by Golbach (1994), nor by Guzmán de Tomé \& Aranda (2008). Currently in Elaterinae, Cardiorhinini

\section{bachmanni [Cardiorhinus]. Golbach}

One spec. pinned, "Misiones" print., remainings of a black frame;"Col. / C. Bruch" print., black frame; "Cardiorhinus [print.] / bachmanni / n. sp. [handwr.]" on white paper; "Holotipo" print. on red paper. "MACN-En / 8735" print., inverted. Ex CBC.

Notes: Probably not published. Not recorded by Golbach (1994), nor by Guzmán de Tomé \& Aranda (2008). Currently in Elaterinae, Cardiorhinini.

\section{consputus [Aeolus]. Schwarz}

One spec. on a card, "Rep. Argentina / Prov. Buenos Aires / 9.VII.1903 / C. Bruch" print., date handwr., black frame; "Typus" print. on pale green paper; "Aeolus / consputus / Type Schw." handwr. by Bruch, red frame.. "MACN-En / 8332" print, inverted.

Notes: Probably not published. Not recorded by Schwarz in Wytsman (1907), Bruch (1911), Schenkling in Schenkling (1925), Blackwelder (1944), nor Golbach (1994). Currently in Agrypninae Oophorini Monocrepidiina. 
flavobasalis [Aeolus]. Schwarz

Two spec. on cards, on one pin, "Rep. Argentina / Prov. Buenos Aires / 9.VII.1903 / C. Bruch" print., date handwr., black frame, "Typus" print. on pale green paper; "Aeolus / flavobasalis / Type Schw." handwr. by Bruch, red frame. "MACN-En / 8331" print., inverted.

Notes: Probably not published. Not recorded by Schwarz in Wytsman (1907), Bruch (1911), Schenkling in Schenkling (1927), nor Blackwelder (1944). Ex CBC. Currently in Agrypninae Oophorini Monocrepidiina.

\section{flavus [Monocrepidius]. Schwarz}

One spec. on a card "Jatahy /Goyaz" handwr.; "Cotypus" print on pale green paper; "Nonocrepidius / flava / Schw.." handwr. by Bruch, red frame, "MACN-En / 8341" print., inverted.

Notes: Probably not published. Not recorded by Schwarz in Whytsman (1907), Bruch (1911), Schenkling in Schenkling (1927), Blackwelder (1944), nor Golbach (1994). Ex CBC. Currently in Agrypninae Oophorini Monocrepidiina.

\section{gracilis $[$ Aeolus]. Schwarz}

Two spec. on cards, on separate pins, "Rep. Argentina / Prov. Tucuman / XI.1899 [190_] / C. Bruch" print., date handwr., black frame; "Typus" print. on pale green paper; "Aeolus / gracilis / Type Schw." handwr. by Bruch, red frame. "MACN-En / 8328 [8329]" print., inverted.

Notes: Not published. Not recorded by Schwarz in Whytsman (1907), Bruch (1911), Schenkling in Schenkling (1927), Blackwelder (1944), nor Golbach (1994). Recorded mistakenly by Guzmán de Tomé \& Aranda (2008: 571). Ex CBC. Currently in Agrypninae Oophorini Monocrepidiina.

\section{niger [Monocrepidius]. Schwarz}

One spec. pinned, "Rep. Argentina / Prov. Buenos Aires / 2.1899 / C. Bruch" print., date handwr., black frame; "Typus" print. on pale green paper; "Monocrepidius / niger / Type Schw." handwr. by Bruch, red frame. "MACN-En / 8342" print., inverted.

Notes: Probably not published. Not recorded by Golbach (1994). Ex CBC. Currently in Agrypninae Oophorini Monocrepidiina.

\section{phaeratus [Aeolus]. Schwarz}

One spec. on a card, "Rep. Argentina / Prov. Tucuman / XII.1899 / C. Bruch" print., date handwr., black frame; "Cotypus" print. on pale green paper; "Aeolus / phaeratus / Schw." handwr. by Bruch, red frame. "MACN-En / 8327" print., inverted.

Notes: Probably not published. Not recorded by Golbach (1994). Ex CBC. Currently in Agrypninae Oophorini Monocrepidiina.

\section{Specimens labeled as types, but probably not conforming a type series}

bruchi (sub Bruchi) [Triplonychus]. Schwarz 1906: 144-145. Tucumán, one spec. from Bruch, first Argentine species of the genus.

One spec. on a card, "Rep. Argentina / Prov. Salta / 190_ / C. Bruch" print., black frame; "Typus" print. on pale green paper; "Horistonotus / Bruchi / type / Schw," handwr. by Bruch, remainings of a black frame. We add "Triplonychus / Bruchi / Schwarz 1906" handwr. on red paper. "MACN-En / 8351" print., inverted.

Notes: Only one spec. from Tucumán was mentioned. Labeled only by Bruch, not by Schwarz. Recorded as Horistonotus Bruchi by Schwarz in Wytsman (1907: 179), by Bruch (1911: 249), by Schenkling in Schenkling (1925: 258) and as Horistonotus bruchi by Golbach (1994: 32). Recorded under Triplonychus by Blackwelder (1947: 303).Ex CBC. Currently in Cardiophorinae Cardiophorini.

drakei (sub Drakei)[Monocrepidius]. Schwarz 1896: 99-100. Paraguay, one $\hat{o}$, one + leg. Drake.

One spec. on a card, "Rep. Argentina / Prov. Tucuman / V.1891 / C. Bruch" print., date handwr., black frame; "Cotypus" print. on pale green paper; "Monocrepidius / Drakei / Schw." handwr. by Bruch, red frame. "MACN-En / 8338" print., inverted.

Notes: spec. compared with lectotype, in coll. Deutsches Entomologisches Institut Eberswalde (Germany), and cited from Argentina and Paraguay by Guzmán deTomé (1998: 301). Only Paraguay was originally mentioned. Recorded under Conoderus by Fleutiaux (1911: 252), Schenkling (1925: 114), Blackwelder (1944: 287) and Golbach (1994: 34 ). Currently in Agrypniinae Oophorini.

flavovittatus (subflavo-vittatus) [Mesembria]. Schwarz 1900: 351. Brasil, ô, ๆ, On page 358 sub flavovittatus in a list

One spec. on a card, "Rep. Argentina / Prov. Buenos Aires / IV.1899 / C. Bruch" print., date handwr., black frame; "Schwarz det." print, 
"Typus" print. on pale green paper; "Cosmesus / flavovittatus / Schw." handwr. by Bruch, red frame. "MACN-En / 8347" print., inverted Notes: Only Brasil was mentioned. Recorded under Mesembria by Arnett (1955: 601), and by Golbach (1994: 38). Cited from Brazil and Argentina by Guzmán de Tomé and Aranda (2008). Ex CBC. Currently in Elaterinae Steatoderini.

pseudoscalaris [Monocrepidius]. Schwarz 1896: 98-99. Paraguay, Drake leg.

One spec. pinned, "Rep. Argentina / Prov. Catamarca / I.1897 / C. Bruch" print., date handwr., black frame; "Cotypus" print. on pale green paper; "Monocrepidius / pseudoscalaris / 15 [16?] / Schw." handwr. by Bruch, red frame. "MACNEn / 8339" print., inverted.

Notes: spec. compared with lectotype in Deutsches Entomologisches Institut, Eberswalde(Germany) and cited from Argentina and Paraguay by Guzmán de Tomé (1998 b: 389). Only Paraguay was mentioned; moreover the collecting date is later than the publishing date. Recorded under Conoderus by Fletiaux (1911: 252), Blackwelder (1944: 288), and Golbach (1994: 35). Ex CBC. Currently in Agrypniinae Oophorini.

\section{BIBLIOGRAPHY}

Arnett, R.H. 1955. Supplement and corrections to J.A. Hyslop's genotypes of the elaterid beetles of the World. Proceedings of the United States National Museum 103 (3336): 599-619.

Arnett, R.H. 1960. Beetles of the United States (A manual for identification): xi +1112 p. Catholic University of America Press, Washington, USA.

Blackwelder, R.E. 1944. Checklist of the coleopterous insects of Mexico, Central America, the West Indies, and South America. Part 2. United States National Museum Bulletin 185 (2): 189-341.

Bouchard, P.; Bousquet Y., Davies, A.E., AlonsoZarazaga, M.A. Lawrence, J.F., Lyal, C.H.C., Newton, A.F., Reid, C.A.M., Schmitt, M.S., Šlipiński, S.A. \& Smith, A.B.T. 2011. Catalogue of the Family-group Names in Coleoptera (Insecta). ZooKeys 88: 1-972 .

Brèthes, J. 1920. Insectes du Pérou. Anales de la Sociedad Científica Argentina 89: 27-54.

Bruch, C. 1911. Catálogo sistemático de los coleópteros de la República Argentina. Pars V. Familias Buprestidae, Trixagidae, Monommidae, Eucnemidae, Elateridae. Revista del Museo de La Plata 17 ( $2^{\text {a }}$ serie 4): 226-260.

Burmeister, H. (1875). Elaterina Argentina. Stettiner Entomologische Zeitung 36 (7/9): 265-273.

Candèze, E.C.A. 1896. Élatérides nouveaux. Sixième fascicule. Mémoires de la Societé Royale des Sciences,
Liège, Belgium $2^{\mathrm{e}}$ sér. 19: 1-88 (Not seen).

Casari, S. 2008. A phylogenetic study of the subtribe Dicrepidiina (Elateridae, Elaterinae, Ampedini). Revista Brasileira de Entomologia 52 (2): 182 -260 .

Costa, C. 1975. Systematics and evolution of the tribes Pyrophorini and Heligmini, with description of Campyloxeninae new subfamily (Coleoptera, Elateridae). Arquivos de Zoologia, São Paulo, Brasil 26 (2): 49-190, f. 1-156, maps 1-12.

Fleutiaux, E. 1911. Revision des Trixagidae, Melasidae et Elateridae (Col.) des Antilles Françaises. Annales de la Société Entomologique de France 80: 235-264.

Golbach, R. 1983. Las especies de los grupos 3 y 4 del género Cardiorhinus Eschscholtz (Col. Elateridae). Acta Zoologica Lilloana Tucumán 37 (1): 153-174, pl. I-III.

Golbach, R. 1984. Duretia, nuevo género de la subfamilia Ampedinae. Acta Zoologica Lilloana Tucumán 38 (1): 41-43.

Golbach, R. 1987. Conoderus argentinus n. sp. (Col. Elat.) y comentarios sobre especies vecinas. Acta Zoologica Lilloana Tucumán 39 (1): 17-21.

Golbach, R. 1987. Cambios en los grupos III y IV del género Cardiorhinus Eschscholtz y descripción de especies nuevas (Col. Elateridae). Acta Zoologica Lilloana Tucumán 39 (1): 29-36 pl. I.

Golbach, R. 1992. Redescripción de Stibadoderus murinus Burm., 1875 (Col. Elat.). Acta Zoologica Lilloana Tucumán 40 (2), 1991: 83-86, f. 1-5.

Golbach, R. 1994. Elateridae (Col.) de la Argentina. Historia, catálogo actualizado hasta 1991 inclusive y clave de subfamilias y de géneros de Centro y Sudamérica. Opera Lilloana 41: 1-48.

Golbach, R. \& Aranda, S. 1991. Athous fueguensis n. sp., primer representante de la subfamilia Athouinae en la Argentina (Col. Elat.). Acta Zoologica Lilloana Tucumán 40 (1): 83-87, f. 1-7.

Guzmán deTomé, M, 1998. Revisión del grupo II del género Conoderus Eschsch. (Coleoptera, Elateridae) de la República Argentina. Descripción de una nueva especie y redescripción de cuatro. Lista y clave de las especies argentinas del grupo II. Acta Zoologica Lilloana 44 (2): 379-398.

Guzmán de Tomé, M. \& Aranda, S. 2008. Familia Elateridae. In: Claps, L.E.; Debandi, G. \& RoigJuñent, S. (Dir.). Biodiversidad de Artrópodos Argentinos 2: 553-563. Ed. Sociedad Entomológica Argentina.

Johnson, P.J. 2002 . 58. Elateridae Leach 1815: 160-173. In: Arnett, R.H. Jr., Thomas, M.C., Skelley, P.E. \& Frank, J.H. (Ed.) American. Beetles. 2. Polyphaga: Scarabaeoidea through Curculionoidea. CRC Press, Boca Raton, Florida, USA: xiv + 861 p.

Lawrence, J.F. 2001. Las familias de Coleoptera de Costa Rica. Familia Elateridae. WWW.inbio.ac.cr /papers/coleoptera/ELATERIDAE

Lawrence, J.F. \& Newton, J.R. 1995. Families and subfamilies of Coleoptera (with selected genera, notes, references and dates on family-group names: 7791098. In Papaluk, J. \& Šlipiński, S.A. (Ed.) Biology, 
Phylogeny, and Classification of Coleoptera. Papers Celebrating the 80th Birthday of Roy Crowson. Warszawa, Muzeum i Instytut Zoologii, Polska Academia Nauk, 2: i-vi + 559-1092.

Rodríguez, D. 2007-2011. Aurora. Aplicación para el manejo de datos de colecciones biológicas. Museo Argentino de Ciencias Naturales "Bernardino Rivadavia", Consejo Nacional de Investigaciones Científicas y Técnicas, CONICET.

Schenkling, S. 1925, 1927. Elateridae.I, II. In Schenkling, S. (Ed.) Coleopterorum Catalogus pars 80: 1-263, 1925; pars 88: 265-639, 1927.

Schwarz, O. 1896. Neue Elateriden-Arten. Deutsche
Entomologische Zeitschrift 1896: 97-104.

Schwarz, O. 1900. Neue exotische Elateriden. Deutsche Entomologische Zeitschrift 1900: 305-358.

Schwarz, O. 1904. Neue Elateriden aus Süd Amerika. I. Teil. II. Teil. Deutsche Entomologische Zeitschrift 1904 (1): 49-69; 70-80.

Schwarz, O. 1906. Neue Elateriden aus Amerika. Deutsche Entomologische Zeitschrift 1906 (1): 97-158.

Schwarz, O. 1907. Coleoptera, familia Elateridae. In Wytsman, P. (Ed.) Genera Insectorum fascic. 46C: 225-370, pl. 1-6.

Recibido: 28-IX-2012

Aceptado: 20-II-2013 